\title{
Osteoid osteoma of the hallux: a case report and review of the literature
}

\author{
Ferrao $\mathrm{PNF}^{1}$, Saragas $\mathrm{NP}^{2}$, Strydom $\mathrm{A}^{3}$, Tladi $\mathrm{M}^{4}$
}

$1 \mathrm{MBChB}$ (Pret); FCS (SA) Ortho; The Orthopaedic Foot and Ankle Unit, Netcare Linksfield Hospital, Johannesburg; Consultant, Department of Orthopaedic Surgery, University of the Witwatersrand, Johannesburg, South Africa

2 MBBCh(Wits); FCS (SA) Ortho; MMed(OrthoSurg)(Wits); The Orthopaedic Foot and Ankle Unit, Netcare Linksfield Hospital, Johannesburg; Professor, Department of Orthopaedic Surgery, University of the Witwatersrand, Johannesburg, South Africa

3 MBBCh(Wits); FCOrth(SA); MMed(OrthoSurg)(Wits); The Orthopaedic Foot and Ankle Unit, Netcare Linksfield Hospital, Johannesburg; Consultant, Department of Orthopaedic Surgery, University of the Witwatersrand, Johannesburg, South Africa

$4 \mathrm{MBChB}($ Limpopo); FCOrth(SA); The Orthopaedic Foot and Ankle Unit, Netcare Linksfield Hospital, Johannesburg; Consultant, Dr George Mukhari Academic Hospital, Sefako Makgatho Health Science University, Ga-Rankuwa, South Africa

Corresponding author: Dr PNF Ferrao, Linksfield Hospital; email: paulo@cybersmart.co.za

\begin{abstract}
Osteoid osteoma $(\mathrm{OO})$ of the phalanges of the foot is a rare occurrence, making it a diagnostic dilemma. Classically OO presents with localised pain, which is worse at night. This pain is often relieved by the use of nonsteroidal anti-inflammatory drugs (NSAID). In the foot however it does not exhibit the classic radiological picture. This makes it difficult to distinguish from a more common diagnosis like infection and inflammation. This diagnosis should be considered in patients with no obvious cause for chronic night foot pain. We present a case of a child with osteoid osteoma of the hallux.
\end{abstract}

Level of evidence: Level 5

Key words: osteoid osteoma, hallux, foot, tumour, night pain

Citation: Ferrao PNF, Saragas NP, Strydom A and Tladi M. Osteoid osteoma of the hallux: a case report and review of the literature. SA Orthop J 2018;17(3):52-56. http://dx.doi.org/10.17159/2309-8309/2018/v17n4a7

Editor: Prof LC Marais, University of KwaZulu-Natal, Durban

Received: June $2017 \quad$ Accepted: December 2017

Published: August 2018

Copyright: ( 2018 Ferrao PNF, et al. This is an open-access article distributed under the terms of the Creative Commons Attribution Licence, which permits unrestricted use, distribution and reproduction in any medium, provided the original author and source are credited.

Funding: No funding was received for this paper.

Conflict of interest: The authors have no conflicts of interest to declare. 


\section{Introduction}

Osteoid osteoma (OO) was first described by Jaffe in $1935 .{ }^{1}$ It is an uncommon osteoblastic lesion of the bone that accounts for about $10 \%$ of all benign bone tumours and $5 \%$ of all primary bone tumours. ${ }^{2,3}$ Osteoid osteoma has not been shown to undergo malignant change but can progress into an osteoblastoma, which is defined as an $\mathrm{OO}$ larger than $15 \mathrm{~mm}^{4}$

$\mathrm{OO}$ is commonly characterised as having a well-demarcated core (nidus) measuring less than $10 \mathrm{~mm}$ in diameter, and a distinctive surrounding zone of reactive bone formation. ${ }^{5}$ The $\mathrm{OO}$ nidus contains vascular osteoid tissue, which may show mineralisation. ${ }^{6}$ This is not the case, however, when presenting in the foot.

It is not commonly found in the foot, less so in the phalanges of the toes. ${ }^{7}$ Despite this lesion not being influenced by trauma, when it occurs in children, parents often give a history of a traumatic episode. The radiographic appearance in the foot is also very atypical. This results in delayed or misdiagnosis of the condition.

The differential diagnosis in these cases includes Garre's osteomyelitis (chronic sclerosing osteomyelitis), Brodie's abscess, stress fracture, tarsal coalition, trauma, osteonecrosis, and inflammatory arthritis. ${ }^{8}$

We describe the case of an $\mathrm{OO}$ involving the left hallux of an 8-year-old girl.

\section{Case study}

An 8-year-old girl presented with a one-year history of left hallux pain. She had visited her general practitioner (GP) a couple of months' prior for this problem. The GP diagnosed her with an ingrown toenail and managed it accordingly. Her symptoms did not resolve and thus the parents sought a second opinion. With regards to the history of her symptoms, the parents first noticed that her left hallux was larger than the right. She then started experiencing intermittent pain, which became progressively more frequent. The pain was worse after her ballet lessons. She would use nonsteroidal anti-inflammatory drugs (NSAIDs) on occasion, which helped significantly with the pain.

On clinical examination she had a normal gait. The left hallux was significantly larger than the right. The skin was erythematous, with no increased local temperature. There was mild tenderness on deep palpation. Range of motion in the metatarsal phalangeal (MP) and interphalangeal (IP) joints was normal with no pain. The foot was neurovascularly intact.

X-rays of the left hallux showed a small oval lytic lesion in the distal phalanx with a central nidus (Figure 1). Blood investigations showed no signs of infection.

CT scan of the left foot demonstrated soft tissue hypertrophy around the left hallux. There was a benign-looking rounded radiolucent lesion with a central calcified nidus measuring $4 \mathrm{~mm}$

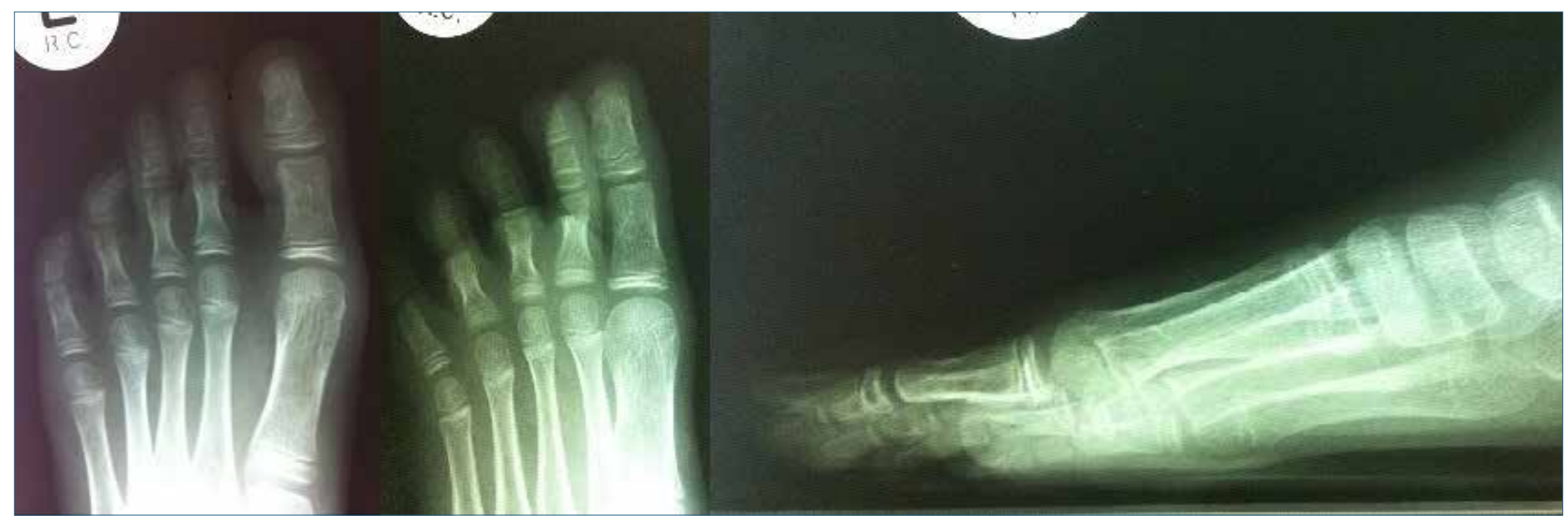

Figure 1. X-rays of the left hallux with a lytic lesion in the distal phalanx with a central nidus
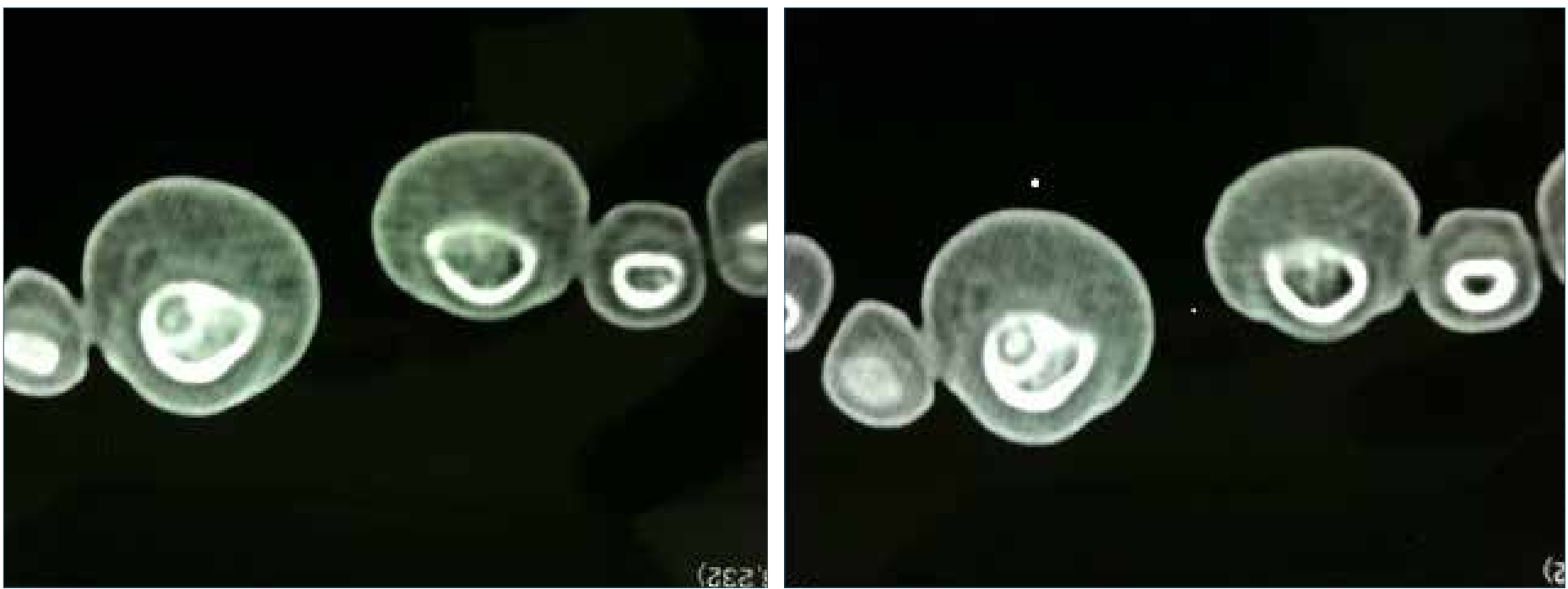

Figure 2. CT scan of the hallux showing a rounded radiolucent lesion with a central calcified nidus 


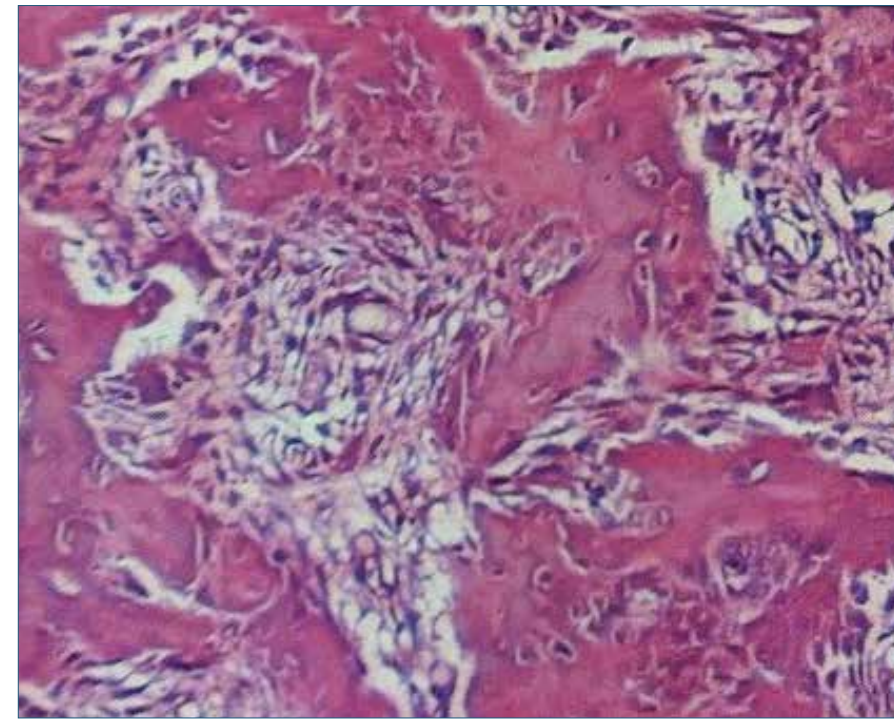

Figure 3. Histological image of the osteoid osteoma

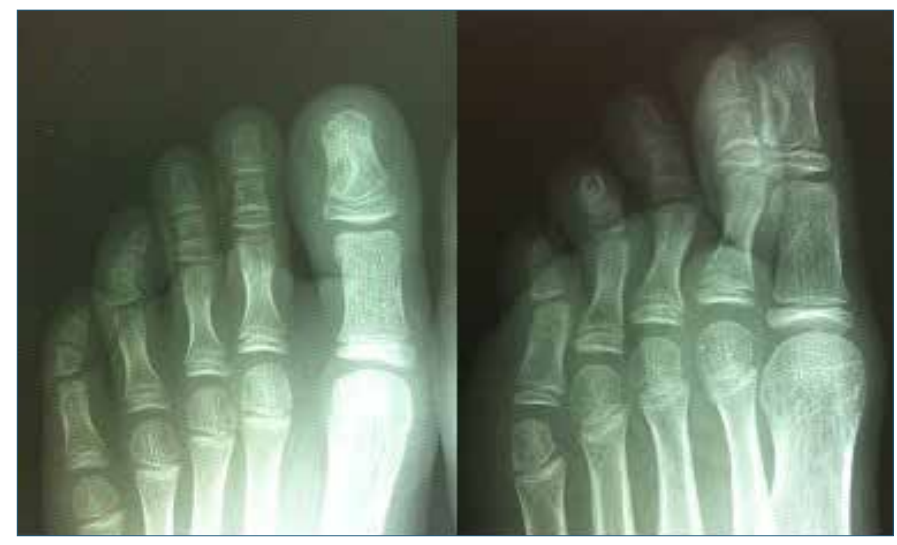

Figure 4. X-rays of the hallux 12 weeks post-surgery showing no central nidus

in the proximal aspect of the diaphysis of the distal phalanx of the hallux. Surrounding bony sclerosis was present (Figure 2).

Consent was obtained for an excisional biopsy of the lesion. The tumour was approached through a dorsolateral incision. The periosteum was found to be normal on inspection. The lesion was identified under image intensifier and curetted out through a small bony window. Specimens were sent for histology. She was managed in a post-operative shoe. Her symptoms resolved dramatically post-operatively.

Histology reported the presence of irregular bone trabeculae, showing a haphazard orientation. Evidence of osteoid formation was noted. There was associated osteoblastic rimming (Figure 3). The diagnosis of $\mathrm{OO}$ was confirmed.

At 6 weeks follow-up she had no symptoms and had resumed ballet. X-rays were repeated at 12 weeks post-surgery, which showed no central nidus (Figure 4).

She was contacted nine years after surgery for review. She reported no recurrence of the symptoms in the left hallux. Currently she performs modern dancing with no discomfort. The left hallux has the same shape and size as the right hallux. Range of motion in the MP and IP joints is normal. There is no tenderness.

Repeat CT scan showed complete resolution of the $\mathrm{OO}$.

\section{Discussion}

Osteoid osteoma is a benign osteogenic tumour measuring less than $15 \mathrm{~mm}$ in diameter; if larger than this it is referred to as an osteoblastoma. ${ }^{1,9}$ It accounts for $10 \%$ of all benign bone tumours. They are commonly found in the long bones of the lower extremity at the meta-diaphyseal region. ${ }^{6}$ More than $50 \%$ occur in the femur and tibia. The proximal femur is the most common site of occurrence. ${ }^{10,11}$ In the foot it mainly occurs in the small bones, with an incidence between $2 \%$ to $11 \%$ (Freiberger et al.). The talus is the most commonly affected small bone in the foot, followed by the calcaneus. ${ }^{7,12}$ The incidence of $\mathrm{OO}$ in the phalanges of the foot is as low as $1-2 \%$ of all foot cases. ${ }^{11}$

It occurs predominantly in children and young adults, ${ }^{2,10}$ with a peak incidence in the second decade. ${ }^{13}$ The male-to-female ratio is reported at about $3: 1 .^{10}$

The classic clinical presentation is that of circumscribed pain that starts off being intermittent and progresses to a constant pain. Frequently the pain is described as being worse at night. ${ }^{8}$ The intensity of the pain is usually not affected by the patient's activity level. ${ }^{14}$ This localised pain is typically relieved by NSAIDs, such as aspirin. This response is often used as part of the diagnostic criteria for $\mathrm{OO}$. The pain caused by these tumours results from the production of large amounts of prostaglandins (PG), which affects this vascular tumour. This is thought to be the reason that NSAIDS are effective in relieving pain in these patients. ${ }^{15}$ For the same reason alcohol, which is a vasodilator, may precipitate an acute pain crisis. ${ }^{8}$ Up to a one thousand-fold rise of PGE2 synthesis has been demonstrated in these tumours. ${ }^{16}$ Schulman et al. identified nerve fibres within the vascular matrix of the nidus in 16 of 18 lesions he studied. They concluded that pain might be mediated by the autonomic nervous system via those fibres, which are sensitive to changes in vascular pressure. ${ }^{17,18}$ These nerve fibres are not seen in an osteoblastoma, and thus used as a histological differentiation from $\mathrm{OO}$. Others feel that the extremely vascular nature of the lesion increases intraosseous tension and oedema, causing pain by direct stimulation of these nerves surrounding the perinidal vessels. ${ }^{19}$

When occurring in the toes, it often causes a fusiform soft tissue swelling and local tenderness, which is atypical for 00 . This enlargement of the toe is difficult to distinguish from digital hypertrophy due to chronic infection or myxoedema. There will often be associated vasomotor disturbances in the overlying skin, resulting in erythema, increased local temperature and sweating. ${ }^{7}$ When in close proximity to a joint referred pain can occur, together with reduced range of motion and adjacent muscle atrophy or spasm. ${ }^{7,13}$ A reactive synovitis and effusion may occur which can simulate arthritis. ${ }^{13,18}$ These lesions mimic inflammatory arthritis or other intra-articular abnormalities. ${ }^{20}$ Clubbing is seen when the lesion occurs in the distal phalanx of the fingers and toes; this is supposedly due to the increased vascularity of this tumour. ${ }^{7}$ In children growth disturbances can occur if the lesion occurs close to an open physis. ${ }^{21,22}$

\section{$X$-rays}

It is classically described as a single oval lytic lesion with a border of reactive sclerosis. ${ }^{10}$ Osteoid osteoma is classified according to its radiographic location, into cortical ( $66 \%-75 \%$ of cases), cancellous and subperiosteal, each with its own distinct radiographic features. ${ }^{10,13}$ The cortical $\mathrm{OO}$ is characterised by a subcortical, $10 \mathrm{~mm}$ in diameter area of radiolucency with dense adjacent bone sclerosis and periosteal reaction. The cancellous type is similar to the cortical but lacks the well-defined sclerotic rim and periosteal reaction. ${ }^{10}$ Bony expansion and endosteal cortical thickening may be present. ${ }^{19}$ The absence of periosteal reaction in intra-articular lesions makes the diagnosis difficult. The subperiosteal 00 presents as a juxtacortical soft-tissue mass eroding the cortex with almost no reactive sclerosis. It can on occasion induce periosteal 
new bone formation. ${ }^{7}$ The cancellous and subperiosteal varieties are mostly prevalent in the small bones of hands and feet, especially the lesser toes. ${ }^{10,11}$ Tomograms can also be helpful, especially in the subperiosteal type.

\section{Computed tomography scans}

Computed tomography (CT) scan is suggested as the investigation of choice for diagnosing OO. Computed tomography visualises the radiolucent low-attenuation nidus with a smooth inner surface of perinidal sclerosis, and the radiodense central calcification. ${ }^{6}$ This central calcification represents the osteoblastic centre of the lesion. ${ }^{10}$ The nidus is best localised with CT slices that are 1 to $2 \mathrm{~mm}$ thick, and the window settings adjusted so that the dense reaction around the lesion does not obscure the small, low-density nidus. ${ }^{8}$

\section{$M R I$}

MRI visualises the nidus as intermediate-to-low signal intensity on T1W SE and intermediate-to-high signal intensity on T2W FSE, PDW or STIR sequences. The lesion is better visualised on T1-weighted images due to the hyperintense marrow oedema found around the nidus. ${ }^{6}$ This bone marrow oedema may help differentiate $\mathrm{OO}$ from inflammatory arthropathy. The difficulty in diagnosing an osteoid osteoma in the foot is that the cancellous bone around the lesion has an increased signal, which can mimic osteomyelitis with a Brodie abscess. ${ }^{14}$ In a recent article, the use of dynamic gadolinium-enhanced MRI has been suggested for the diagnosis of $\mathrm{OO}$ in atypical locations. Davies et al. showed that $35 \%$ of $\mathrm{OO}$ tumours could be missed if investigated by MRI alone. ${ }^{6}$ Clarke et al. showed that in the absence of an identifiable nidus and the presence of oedema localised to a specific bone together with soft tissue oedema, OO should be the primary differential and investigated further with a computed tomography (CT) scan. ${ }^{5} \mathrm{MRI}$ is especially difficult to interpret in subperiosteal $\mathrm{OO}$ due to the extensive soft tissue oedema.

\section{Bone scan}

Bone scan is an investigation which could help identify the 00 . Due to its osteoblastic nature there is an intense uptake of the radionuclide marker by the lesion. This shows up as a typical 'hot spot' on a bone scan. ${ }^{10}$

\section{Pathology}

The macroscopic appearance of the lesion is a small, round or oval, mass of tissue, that can be soft, rubbery or granular in texture. It is red or brownish in colour, which can change to yellowish-white in the presence of calcification. The lesion can be easily removed from its osseous bed. ${ }^{7}$ It contains a variable amount of ossification with a zone of reactive sclerosis. ${ }^{23}$ Histologic examination shows an interlacing network of osteoid trabeculae within vascular fibrous connective tissue, accompanied by benign multinucleated giant cells, and well-differentiated osteoblasts. ${ }^{7,10}$ This makes up the radiolucent zone around the nidus. The perinidal sclerotic zone consists of mineralised bone with either dense cortical bone in the cortical type $\mathrm{OO}$, thickened trabecular bone in the cancellous type $\mathrm{OO}$, or abundant localised periosteal new bone formation in the subperiosteal osteoid type OO.,

\section{Treatment}

A detailed history from the patient and a high index of clinical suspicion are needed by the treating physician in order to correctly diagnose this condition and manage it accordingly without too much delay. ${ }^{10}$

There is literature describing conservative management of $\mathrm{OO}$. This consists mainly of symptomatic relief with NSAIDs. Osteoid osteoma has been shown to resolve after a mean period of 3-4 years. However most $\mathrm{OO}$ tumours are removed within 1 to 2 years due to the progressive intensity of pain and associated symptoms. ${ }^{24}$ As a result, the natural history of untreated $\mathrm{OO}$ is only partially known. ${ }^{14,15}$

Surgical management consists of open resection with intralesional, marginal or wide surgical margins. ${ }^{8,18}$ However, en bloc excision is advised. Surgical excision has the advantages of allowing histological examination and confirmation so as to ensure complete excision. In appendicular lesions where the lesion is within $15 \mathrm{~mm}$ of the neurovascular bundle, surgical excision is preferred. Most patients describe immediate relief of symptoms directly after surgery. Recurrence is rare if the lesion is completely removed. ${ }^{11,25}$ Intra-operative bone scan can be used to assess if the lesion has been removed. Radioisotope is administered to the patient 4 hours prior to surgery. The removed lesion is then screened in theatre; if correct, the excised bone will appear 'hot'. 2,4,13,26,27 Bone graft can be used to fill the defect, but is not usually necessary due to the small size of the lesion.

Modern management options consist of CT-guided percutaneous treatment, with both radiofrequency ablation or laser photocoagulation. The benefits of these techniques are that they are minimally invasive, safe and effective ${ }^{6,28}$ Osteoid osteoma can be very difficult to localise in surgery and thus has made this method increasingly favourable. ${ }^{29} \mathrm{~A}$ CT-guided biopsy is performed first to confirm diagnosis. The use of radiofrequency ablation for the treatment of $\mathrm{OO}$ was first described by Rosenthal et al. ${ }^{30}$ The technique consists of using high frequency alternating current at $500000 \mathrm{~Hz}$, transmitted through a special probe into the lesion under CT guidance. This induces local ionic agitation and frictional heat in the lesion causing coagulation necrosis. Bone loss is kept to a minimum preventing significant structural weakening. ${ }^{18}$ This is particularly useful in juxta-articular foot lesions. Complications associated with radiofrequency ablation are recurrence and damage to neighbouring neurovascular structures, particularly if ablating cancellous bone. ${ }^{11}$

\section{Conclusion}

Osteoid osteoma is a benign tumour occurring mainly in the long bones of the lower limb. It is classically described radiographically as a radiolucent nidus smaller than $15 \mathrm{~mm}$ with surrounding cortical bone. This is true for the common cortical OO. The less common cancellous and subperiosteal types do not have the classic sclerotic border. These two types are commonly found in the foot. This variable clinical and radiological picture makes early diagnosis difficult. Juxta-articular $\mathrm{OO}$ causes referred joint pain resembling arthralgia. The treating physician requires a high index of suspicion, and a CT scan should be ordered if suspected so as not to delay appropriate management. Management consists of surgical excision or newer, less invasive CT-guided ablation. Histopathological confirmation should be obtained on all suspected lesions. Adequate resection results in complete resolution. 


\section{Ethics statement}

Permission was obtained from the patient's parents to present this case report.

\section{References}

1. Jaffe HL. Osteoid osteoma. Arch Surg. 1935;31(5):709.

2. Barca F, Acciaro AL, Recchioni MD. Osteoid osteoma of the phalanx: enlargement of the toe - two case reports. Foot \& Ankle International. 1998;19(6):388-93.

3. Schajowicz F. Histological classification of bone tumours. In Histological typing of bone tumours. Springer Science + Business Media; 1993. p. 3-6.

4. Gitelis S, Schajowicz F. Osteoid osteoma and osteoblastoma. The Orthopedic Clinics of North America. 1989;20(3):313-25.

5. Anderson WAD. Pathology. St Louis: CV Mosby. 1977. pp1970-2011.

6. Shukla S, Clarke AW, Saifuddin A. Imaging features of foot osteoid osteoma. Skeletal Radiol. 2009;39(7):683-89.

7. Sproule JA, Khan F, Fogarty EE. Osteoid osteoma: painful enlargement of the second toe. Archives of Orthopaedic and Trauma Surgery. 2004;124(5):354-56.

8. Morbidi M, Ventura A, Della Rocca C. Arthroscopic assisted resection of juxta-articular osteoid osteoma. The Journal of Foot and Ankle Surgery. 2007;46(6):470-73.

9. Patnoe TG, Pritchard DJ. Benign osteoblastoma. Orthopedics. 1989;12(2):329-31.

10. Deveci A, Delialioglu OM, Daglar B, Tunç SC, Birinci B, Bayrakci K et al. An unusual cause of forefoot pain: diagnosis and discussion. Skeletal Radiol. 2009;38(8):831-32.

11. Jowett CRJ, Singh D. Osteoid osteoma of the great toe: a case report. Foot and Ankle Surgery. 2010;16(2):e12-e5.

12. Jay RM. Surgical treatment of osteoid osteoma in an adolescent The Journal of Foot Surgery. 1990;29(5):495-98.

13. Chang JL, Ireland ML. Osteoid osteoma of the os calcis in a teenage athlete. Medicine and Science in Sports and Exercise. 1993;25(1):2-8.

14. Morris GB, Goldman FD. Osteoid osteoma causing subtalar joint arthralgia: a case report. The Journal of Foot and Ankle Surgery. 2003;42(2):90-94.

15. Campanacci M, Ruggieri P, Gasbarrini A, Ferraro A, Campanacci L. Osteoid osteoma. The Journal of Bone and Joint Surgery. 1999;81(5):814-20.

16. McCarten G, Dixon P, Marshall D. Osteoid osteoma of the distal phalanx: a case report. The Journal of Hand Surgery: Journal of the British Society for Surgery of the Hand. 1987;12(3):391-93.

17. Schulman L, Dorfman HD. Nerve fibers in osteoid osteoma The Journal of Bone and Joint Surgery American volume. 1970;52(7):1351-56.

18. Migues A, Velan O, Solari G, Pace G, Slullitel G, Araujo ES. Osteoid osteoma of the calcaneus: percutaneous radiofrequency ablation. The Journal of Foot and Ankle Surgery. 2005;44(6):469-72.

19. Ebrahimzadeh MH, Omidi-Kashani F, Hoseini MR. Painful and tender toe, osteoid osteoma of the distal phalanx of toe, a diagnostic dilemma. The Foot. 2009;19(4):232-35.

20. Snarr JW, Abell MR, Martel W. Lymphofollicular synovitis with osteoid osteoma 1. Radiology. 1973;106(3):557-60.

21. O'Connell JX, Nanthakumar SS, Nielsen GP, Rosenberg AE. Osteoid osteoma: the uniquely innervated bone tumor. Modern Pathology: an official journal of the United States and Canadian Academy of Pathology, Inc. 1998;11(2):175-80.

22. Spencer EE, Beirman JS, Femino JE. Osteoid osteoma of the fifth metatarsal: a case report and literature review. Foot and Ankle Surgery. 2002;8(1):71-78.

23. Resnick D KM, Greenway GD. Diagnosis of bone and joint disorders. Saunders; 1995.

24. Voutsinas S, Tillianakis M, Tsouparopoulos D. Osteoid osteoma producing hypertrophy of a toe. The Foot. 1991;1(1):35-36.
25. Shereff MJ, Cullivan WT, Johnson KA. Osteoid-osteoma of the foot. The Journal of Bone and Joint Surgery American volume. 1983;65(5):638-41.

26. LaCroix ML, Thomas JR, Nicholas RW. Subperiosteal osteoid osteoma of the distal phalanx of the fourth toe. Orthopedics. 2001;24(7):695-96.

27. Öztürk A, Yalçınkaya U, Özkan Y, Yalçın N. Subperiosteal osteoid osteoma in the hallux of a 9-year-old female. The Journal of Foot and Ankle Surgery. 2008;47(6):579-82.

28. Zouari L, Bousson V, Hamze B, Roulot E, Roqueplan F, Laredo JD. CT-guided percutaneous laser photocoagulation of osteoid osteomas of the hands and feet. European Radiology. 2008;18(11):2635-41.

29. Cantwell CP, Obyrne J, Eustace S. Current trends in treatment of osteoid osteoma with an emphasis on radiofrequency ablation. European Radiology. 2004;14(4):607-17.

30. Rosenthal DI, Alexander A, Rosenberg AE, Springfield D. Ablation of osteoid osteomas with a percutaneously placed electrode: a new procedure. Radiology. 1992;183(1):29-33. 